

\title{
Correlation between Prostate Specific Antigen and Prostate Biopsy Gleason Score
} Ngwu PE ${ }^{* 1}$, Achor GO1,2, Eziefule VU1, Orji JI' ${ }^{1}$, Alozie FT ${ }^{1}$

1Division of Urology, Department of Surgery, Federal Medical Centre, Umuahia, Nigeria ${ }^{2}$ Division of Urology, Department Of Surgery, Abia State University Teaching Hospital, Aba, Nigeria

*Correspondence: Dr. PE Ngwu, Department Of Surgery, Federal Medical Centre, Umuahia, Nigeria. Email: paulngwu66@gmail.com; ORCID - https://orcid.org/0000-0002-9869-5079

\begin{abstract}
Background: Prostate Specific Antigen (PSA) is a commonly used marker in prostate cancer management. Gleason grading is one of the most powerful predictors of prostatic biological behaviour. PSA, when combined with the Gleason score and clinical stage, improves the prediction of the pathological stage for prostate cancer.

Objectives: To assess the degree of correlation between PSA level and Gleason score as well as determine the likelihood of aggressiveness of prostate cancer using Gleason score as a parameter.

Methods: A cross-sectional prospective study was conducted among 234 consecutive consenting patients presenting to the Urology Out-Patient Clinic between April 2015 and March 2018. Serum PSA was done and patients with values above $4 \mathrm{ng} / \mathrm{ml}$ and/or abnormal Digital Rectal Examination (DRE) were selected to have a prostate biopsy. The sample was histologically analysed with Gleason score recorded for those with prostate cancer. Gleason score was then correlated with PSA levels.

Results: The mean age for prostate cancer patients was $71.3 \pm 8.7$ years. The mean PSA for patients with prostate cancer was $52.3 \pm 37.5 \mathrm{ng} / \mathrm{ml}$ (Confidence Interval $=46.1-58.6$ ) with $\mathrm{p}<0.001$. About $18.2 \%$ of histologically confirmed prostate cancer cases had Gleason score 8-10 implying a high level of tumour aggressiveness. There is a positive correlation between PSA and Gleason score with R-value 0.590 indicating a good degree of correlation.

Conclusion: There is a good degree of a positive correlation between PSA level and Gleason score, as well as a high level of aggressiveness of prostate cancer in Umuahia.
\end{abstract}

Keywords: Digital rectal examination, Gleason score, Histological Analysis, Prostate cancer, Prostate Specific Antigen.

\section{Introduction}

Prostate cancer is the most common male genital cancer. [1] Various studies in Nigeria have shown varying but relatively high incidence rates among Nigerians. [2,3] Prostate Specific Antigen (PSA) is a serine protease produced by prostatic epithelium and peri-urethral glands. [4] Although a controversial screening tool, PSA remains a useful parameter for monitoring treatment. [5]

There are few systems used for the estimation of tumour cells differentiation in the evaluation of Carcinoma of the Prostate $(\mathrm{CaP})$ and the Gleason scoring system is the most widely used. 
[6] Where it is established that there is a correlation between PSA and the Gleason score, the implication will be that the higher the PSA, the higher the chances that the patient will have a high Gleason score. Since it is widely accepted that the higher the Gleason score, the poorer the prognosis, this will guide the Urologist in advising his patients on how pressing the need for a prostate needle biopsy is, to confirm the diagnosis of $\mathrm{CaP}$. Therefore, this study was designed to assess the degree of correlation between the Prostate Specific Antigen and the Gleason score in CaP.

\section{Methods}

This was a prospective, cross-sectional, single centre study, carried out between April 2015 and March 2018. The study location is a hospital that runs a busy Urology Out-Patient Clinic that operates twice a week with an average daily attendance of 60 patients.

All the consecutively consenting patients who were aged $\geq 40$ years, had a serum PSA $>4 \mathrm{ng} / \mathrm{ml}$ or an abnormal digital rectal examination (DRE) were selected for trans-rectal ultrasound (TRUS)-guided prostate needle biopsy. Patients who were already on treatment for $\mathrm{CaP}$ or benign prostatic hyperplasia $(\mathrm{BPH})$ were excluded from the study. Informed consent was obtained from each patient recruited into the study. Ethical clearance was obtained from the Federal Medical Centre, Umuahia (Certificate Number FMC/QEH/G.596/Vol.10/065).

The minimum sample size for the study was calculated as 220 from the formula $n=Z^{2} P q / d 2$ were $\mathrm{Z}$ is the standard normal deviation, usually set at 1.96 which corresponds to the 95 percent confidence interval and $\mathrm{P}$ which is the proportion of the study population estimated to have elevated PSA with histological diagnosis of $\mathrm{CaP}$. A prevalence rate of $17 \%$ as previously reported in a study conducted at Benin City, Nigeria ${ }^{[7]}$ was adopted.

The demographic parameters of the patients were recorded. The serum PSA was analysed using the ELISA method (Strip Reader) manufactured by Monoband ${ }^{\circledR}$ Incorporated USA. Prophylactic antibiotics were administered for each patient. Caudal anaesthesia was given and a 10-13 core transrectal ultrasound-guided, transrectal prostate biopsy was done using an automatic biopsy needle (16G). The patients on anticoagulants and/or NSAIDs were asked to discontinue such therapies for at least one week before the procedure. Histopathological analysis of the biopsy samples was done by the same pathologist. Cancers detected in the biopsy cores were graded histologically according to the Gleason system using the following Gleason categories:

- Well Differentiated - Gleason score 4 and 5

- Moderately Differentiated- Gleason scores 6 and 7

- Poorly Differentiated - Gleason score 8

- Anaplastic - Gleason scores 9 and 10

The data were analysed using the Statistical Package for Social Sciences (SPSS) ${ }^{\circledR}$ version 21.0 for windows. Simple descriptive statistics were used to characterize the socio-demographic and clinical parameters. The confidence interval was set at $95 \%$ and $P$ - values less than 0.05 were considered significant.

\section{Results}

A total of 234 men were recruited out of which, $138(58.9 \%)$ had CaP. The age range of the subjects with CaP was $52-92$ years with a mean of $71.3 \pm 8.7$ years. Figure 1 shows that the $71-80$ years age group had the highest frequency followed by the 61-70years age group. In this cohort, $18.2 \%$ of the patients with histologically confirmed $\mathrm{CaP}$ had aggressive tumours (Figure 
2). These were the patients with anaplastic tumour, corresponding to Gleason score 9 and 10. Figure 3 showed that the median PSA as depicted in the boxplots increased from the moderately differentiated Gleason category to the anaplastic category with $\mathrm{R}$ and $\mathrm{R}^{2}$ values for this model as 0.590 and 0.348 respectively. The $\mathrm{R}$-value is the simple correlation coefficient and the value of 0.590 indicated a good degree of correlation between PSA and Gleason score using Pearson's correlation $(p=0.01)$. The R2 value depicts how much of the total variation in the dependent variable (Gleason score) can be explained by the independent variable (PSA). In this study, the $\mathrm{R}^{2}$ value of 0.348 translated to $34.8 \%$. The pattern of linear correlation between PSA and the Gleason score is shown in Figure 5.

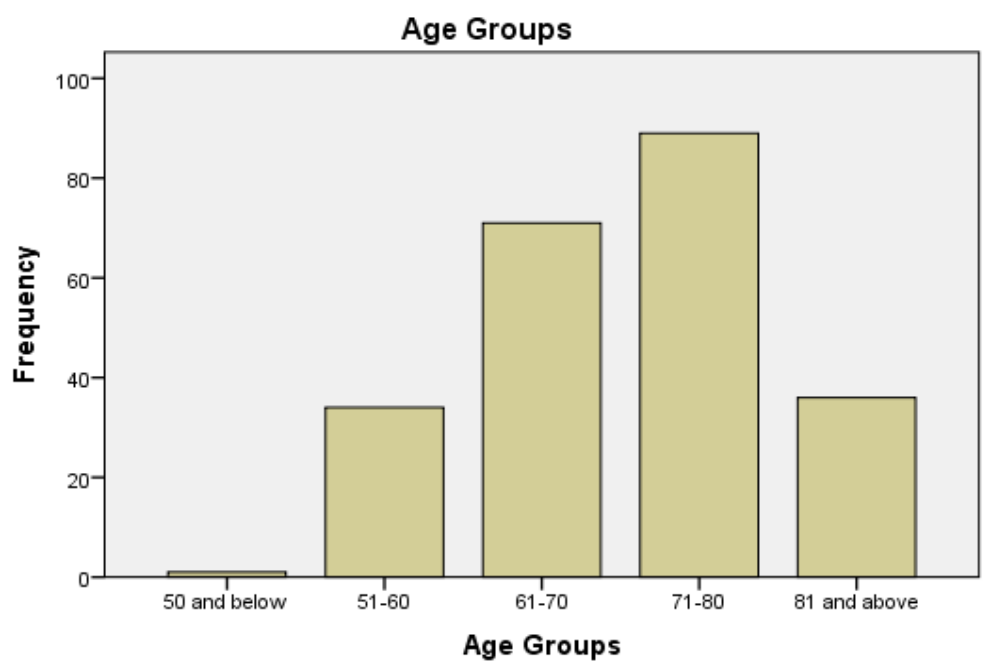

Figure 1: Frequency distribution of the various age groups in the study

\section{Discussion}

This work reported the correlation between PSA and Gleason score and determined the likelihood of aggressiveness of prostate cancer using the Gleason score as a parameter. The age range of 52-92 years with a mean age of 71.3years was similar to the report of the age range of 60-79 years with a mean of 69.9 years from Serbia and Montenegro. ${ }^{[8]}$

The study revealed that $18.2 \%$ of the patients with histologically confirmed $\mathrm{CaP}$ have an aggressive tumour, that is, those in the anaplastic (undifferentiated) cancer group with Gleason score 9 and 10 while poorly differentiated cancer (Gleason score 8) accounted for $29.5 \%$. In a study by Thompson et al., ${ }^{[9]}$ only $14.9 \%$ of men with CaP had a Gleason score of 7 and above. Studies have also shown that men with a Gleason score of 7-10 were between $29 \%$ and $43 \%$ at risk of death from CaP, even when the cancer was diagnosed as late as 74 years and was treated surgically. ${ }^{[10]}$

Since the Gleason score closely correlates with clinical behaviour and also provides an important index of prognosis, [11] it can, therefore, be stated that a significant proportion of the patients with $\mathrm{CaP}$ patients in this study presented with aggressive tumours. The study showed that there is a positive and good correlation between PSA and the Gleason score. 


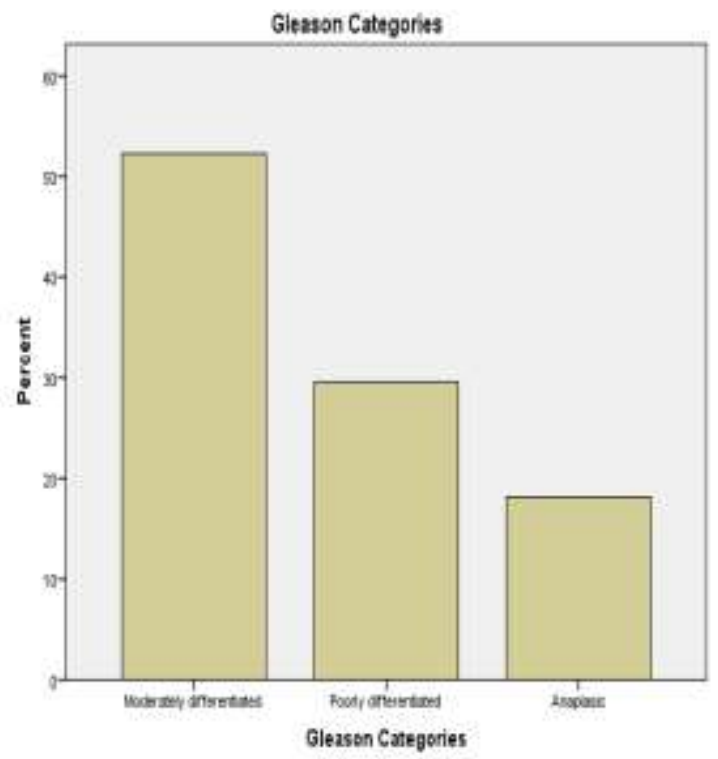

Figure 2: Percentage distribution of CaP cases according to the Gleason Categories

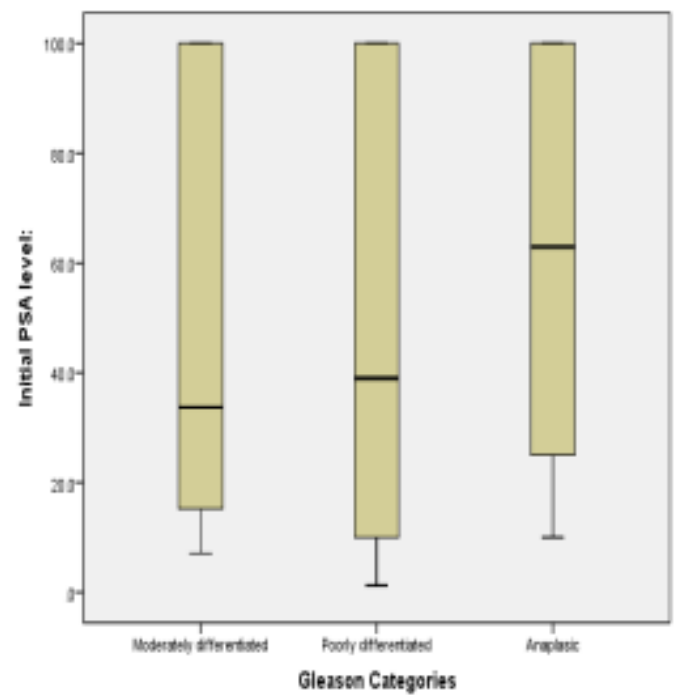

Figure 3: Distributions of PSA values according to the Gleason Score categories among men with Prostate Cancer

This is similar to the finding reported by Zivkovic et al., in Serbia and Montenegro, where a highly significant positive correlation between PSA and Gleason score was observed with an Rvalue of $0.462 .{ }^{[8]}$ A study by Gurumurthy et al. in India also reported a positive correlation between the two parameters. [12] Meanwhile, certain studies reported the fact that serum PSA did not have a strong correlation with the Gleason score. This observation could be explained by the fact that less differentiated tumours, sometimes produce less PSA. This could be explained by the loss of phenotypic expression of PSA, following the dedifferentiation of tumour cells. [13] 


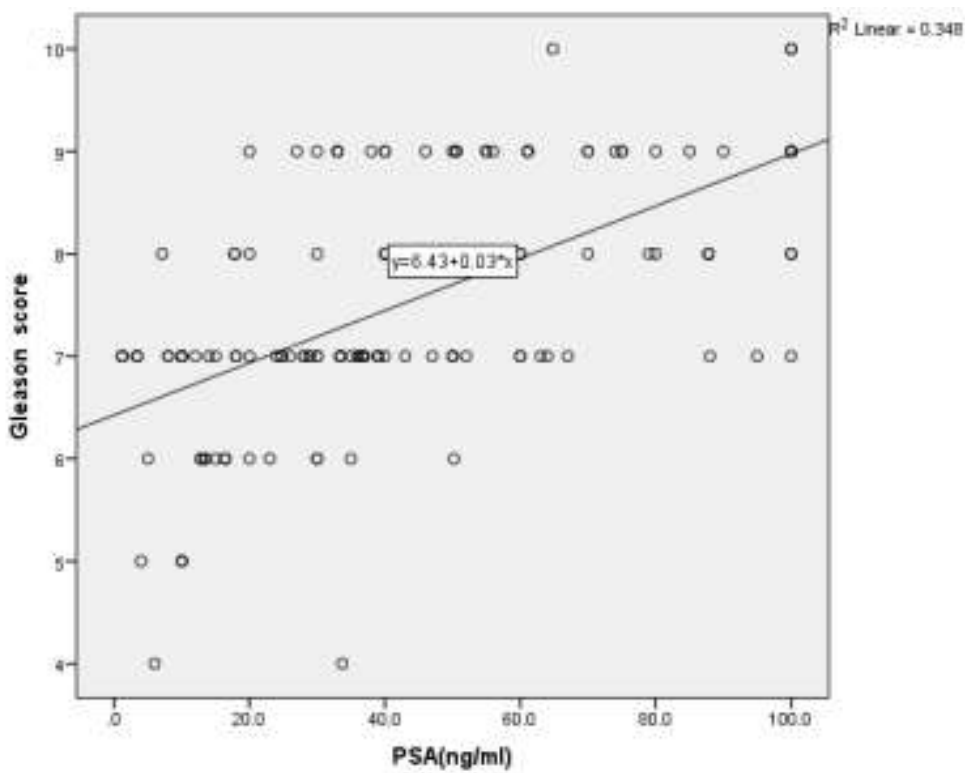

Figure 5: Linear regression line and a coefficient of linear correlation between PSA (ng/ml) and the Gleason score

From the foregoing, the significant correlation between PSA and the Gleason score can be used by urologists to infer that the higher the PSA in the absence of other factors that influence the PSA level, the higher the chances that a patient will have a higher Gleason's prognostic grade of the tumour. This may assist in terms of decision making on when to recommend prostate needle biopsy to patients.

Since the study has shown that there is a positive correlation between PSA and the Gleason score as well as a likely significant relationship with poorly differentiated and anaplastic tumours, there is a need to create more awareness among men. This will enhance the early diagnosis of likely cases of prostatic tumour. However, multi-centre studies are needed in this region as well as in the entire country, to throw more light on this area of research interest, because there is a high incidence as well as morbidity [1,14] associated with $\mathrm{CaP}$ among men of black African descent compared with other races where more extensive collaborative work on the condition has been done.

\section{Conclusion}

The study showed that there is a positive correlation between PSA and the Gleason score. Since the study also showed a significant proportion of cases of $\mathrm{CaP}$ was aggressive, a high index of suspicion as well as strategies to increase patients' awareness, are very important for early detection.

Authors' Contributions: NPE and AGO conceived and designed the study. OJI and AFT participated in data acquisition and analysis while EVU and NPE interpreted the data and participated in drafting the manuscript. AGO and EVU revised the draft manuscript for sound intellectual content. All the authors approved the final draft of the manuscript.

Conflict of Interests: None

Funding: Self-Funded

Publication History: Submitted 31 May 2019; Revised 09 August 2019; Accepted 10 September 2019.

\section{References}

1. Pernar C, Ebot E, Wilson K, Mucci L. The epidemiology of prostate cancer. Cold 
Spring Harb Perspect Med 2018; 8(12): a030361.

2. Ikwuerowo S, Omisanjo O, Bioku M, Ajala M, Mordi V, Esho J. Prevalence and characteristics of prostate cancer among participants of a community-based screening in Nigeria using serum prostate specific antigen and digital rectal examination. Pan Afr Med J 2013; 15: 129.

3. Obiora C, Nwosu S. A histopathological study of carcinoma of the prostate in PortHarcourt, Nigeria. Niger J Clin Prac 2011; 14(3): 363-367.

4. Han M, Gann PH, Catalona WJ. Prostatespecific antigen and screening for prostate cancer. Med Clin N Am 2004; 88: 245-265.

5. Emokpae MA, Das SC, Orok T, Mohammed AZ, Al Hassan S. Early detection of prostate cancer: Evaluating the diagnostic performance of prostate specific antigen by comparing with histological technique among Africans. Indian J Clin Chem 2004; 19(1): 62-66.

6. Chen N, Zhou Q. The evolving Gleason grading system. Chinese J Res 2016; 28(1) 58-64.

7. Akang EE, Aligbe JU, Olisa EG. Prostatic tumours in Benin City, Nigeria. West Afr J Med 1996; 15(1):56-60.
8. Zivkovic S. Correlation between prostate specific antigen and histopathological difference of prostate carcinoma. Arch Oncol 2004; 12(3): 148-151.

9. Sweat SD, Bergstralh EJ, Slezak J, Blute ML, Eincke H. Competing risk analysis after radical prostatectomy for clinically nonmetastatic prostate adenocarcinoma according to clinical Gleason score and patient age. J Urol 2002; 168; 525-529.

10. Graham J, Baker M, Macbeth F, Titshall V. Diagnosis and treatment of prostate cancer: summary of NICE guidance. BMJ (Clinical Research Ed) 2008; 336(7644): 610-612.

11. Gurumurthy D, Maggad R, Patel S. Prostate carcinoma: correlation of histopathology with serum prostate specific antigen. Sci J Clin Med 2015; 4(4): 1-5.

12. Schroder FH, Van-Der Crvijsen-Koeter I, De Koning HJ, Vis AN, Hoedemaeker RF, et al. Prostate cancer detection at low prostatespecific antigen. J Urol 2000; 163(3): 806-812.

13. Dawam D, Rafindadi AH, Kakayi GD. Benign prostatic hyperplasia and prostatic carcinoma in native Africans. Br J Urol 2000; 85: 1074 .

14. Ekwere PD, Egbe SN. The changing pattern of prostate cancer in Nigerians: current status in the southeastern states. J Natl Med Assoc 2002; 94(7): 619-627.

\begin{tabular}{|c|c|c|c|} 
This is an Open Access document licensed for distribution under the terms and conditions of the Creative \\
Commons Attribution License (http://creativecommons.org/licenses/by-nc/4.0). This permits unrestricted, \\
non-commercial use, reproduction and distribution in any medium provided the original source is adequately \\
cited and credited.
\end{tabular}

